ANNALES

POLONICI MATHEMATICI

$95.2(2009)$

\title{
Borel summable solutions of the Burgers equation
}

\author{
by GRZEGorz Łysik (Warszawa)
}

\begin{abstract}
We give necessary and sufficient conditions for the formal power series solutions to the initial value problem for the Burgers equation $\partial_{t} u-\partial_{x}^{2} u=\partial_{x}\left(u^{2}\right)$ to be convergent or Borel summable.
\end{abstract}

1. Introduction. We consider the initial value problem for the Burgers equation

$$
\left\{\begin{array}{l}
\partial_{t} u-\partial_{x}^{2} u=\partial_{x}\left(u^{2}\right), \\
\left.u\right|_{t=0}=u_{0} .
\end{array}\right.
$$

The equation was proposed by J. M. Burgers [6] as a simple model for the statistical theory of turbulence in hydrodynamics. It illustrates the interaction between nonlinear convection and diffusion. There is a vast literature relating to different aspects of the Burgers equation. It is surveyed in [5], where also some explicit solutions can be found. For later references see [12] and [19]. In particular, local in time existence and uniqueness of classical solutions of (1) was first established by E. Hopf [11] under the assumption that $u_{0}$ is a continuous function of linear growth at $\pm \infty$. The IVP (1) was also studied by D. B. Dix [7] who established local well-posedness of (1) in the class $C\left([0, T), H^{s}\right)$ for $s>-1 / 2$. His result was extended by D. Bekiranov [4] to the case of a generalized Burgers equation in the class of weighted $L^{p}$ based Sobolev spaces.

One of the methods in the study of parabolic equations is to look for solutions in the form of formal power series in the $t$ variable, i.e.

$$
\widehat{u}(t, x)=\sum_{k=0}^{\infty} u_{k}(x) t^{k},
$$

and then apply the Borel resummation technique to obtain genuine solutions. The method works well in the case of linear equations. Let us mention

2000 Mathematics Subject Classification: Primary 35C10, 35C20; Secondary 35K55.

Key words and phrases: Burgers equation, analytic solutions, Borel summability. 
here the papers of D. A. Lutz, M. Miyake and R. Schäfke [14], W. Balser and S. Malek [3] and S. Michalik [16], where results on Borel summability of formal solutions of the linear heat equation were established. W. Balser [1], H. Chen, Z. Luo and C. Zhang [8], H. Chen and Z. Zhang [9] and S. Ouchi [17] obtained some results on summability of formal solutions to other linear partial differential equations. For nonlinear equations the situation is more complicated and very few results are known. To our knowledge only S. Ouchi [18] studied formal solutions of some totally characteristic nonlinear partial differential equations and proved their multisummability under the assumption that the initial data vanish on $\{t=0\}$.

Here we assume that the initial data $u_{0}$ is an analytic function on a domain $\Omega \subset \mathbb{R}$ and we are interested in the solutions of (1) in the form of a formal power series (2). Clearly, the formal solution (2) of (1) exists and is unique. Namely, inserting (2) into (1) we obtain the recurrence relations

$$
u_{k+1}=\frac{1}{k+1}\left(\partial^{2} u_{k}+v_{k}\right), \quad k \in \mathbb{N}_{0},
$$

with a given $u_{0}$ and

$$
v_{k}=\sum_{\kappa \in \mathbb{N}_{0}^{2}, \kappa_{1}+\kappa_{2}=k} \partial\left(u_{\kappa_{1}} u_{\kappa_{2}}\right), \quad k \in \mathbb{N}_{0} .
$$

Note that the functions $u_{k}$ are analytic on $\Omega$. In [15] we have proved that the formal power series solution (2) of (1) belongs to the Gevrey class $G^{2}(\Omega)$ in time, i.e. for any compact subset $K \Subset \Omega$ one can find $C<\infty$ such that $\left|u_{k}(x)\right| \leq C^{k+1} k$ ! for $k \in \mathbb{N}_{0}$ and $x \in K$, which is equivalent to convergence of the Borel transform of $\widehat{u}$. Here we give necessary and sufficient conditions for the convergence or Borel summability of formal solutions. Roughly speaking, our results can be formulated as follows (see Theorems 1, 2 and Corollary 2 for precise formulations).

THEOREM. Let $u_{0}$ be an analytic function on a domain $\Omega \subset \mathbb{R}$ containing the origin and $\theta \in \mathbb{R}$.

The formal power series solution (2) of (1) is locally uniformly convergent in $\Omega$ if and only if $u_{0}$ extends to a meromorphic function on $\mathbb{C}$ with at most simple poles with residues in $\mathbb{N}$ and at most linear growth at $\infty$.

The formal power series solution (2) of (1) is Borel summable in direction $\theta \in \mathbb{R}$ if and only if the function $\varphi(x)=\exp \left\{\int_{0}^{x} u_{0}(y) d y\right\}$ extends to a function holomorphic on a domain $D(\theta, \alpha)$ containing a double cone $S(\theta / 2, \alpha) \cup S(\theta / 2+\pi, \alpha)$ with some $\alpha>0$ and has in $D(\theta, \alpha)$ at most an exponential growth of order at most 2.

Our treatment heavily depends on the Cole-Hopf transformation which transforms solutions of the Burgers equation to those of the heat equation. Then we apply a characterization of convergent (resp. Borel summable) so- 
lutions of the heat equation, Borel-Hadamard theorems and properties of the logarithmic derivative.

2. Analytic solutions. In this section we give a characterization of convergent solutions of the Burgers equation. First of all, recall the ColeHopf lemma. Its detailed proof can be found in [11].

Lemma 1. If $\varphi=\varphi(t, x) \in C^{1,3}$ is a solution of the heat equation $\varphi_{t}^{\prime}=$ $\varphi_{x x}^{\prime \prime}$, then its logarithmic derivative

$$
u(t, x)=(\ln \varphi(t, x))_{x}^{\prime}=\frac{\varphi_{x}^{\prime}(t, x)}{\varphi(t, x)}
$$

is a classical solution of the Burgers equation in a domain where $\varphi(t, x) \neq 0$. Conversely, if $u$ is a classical solution of the Burgers equation, then

$$
\varphi(t, x)=\exp \left\{\int_{\grave{x}}^{x} u(t, y) d y\right\}
$$

belongs to $C^{1,3}$ and solves the heat equation.

The mapping $u \mapsto \varphi$ is called the Cole-Hopf transformation.

Note that the logarithmic derivatives of two functions $\varphi_{1}$ and $\varphi_{2}$ coincide if and only if $\varphi_{1}$ and $\varphi_{2}$ differ at most by a multiplicative constant. In the case of the Cole-Hopf transformation the multiplicative constant is recovered by choosing $\stackrel{\circ}{x}$ in (6).

THEOREM 1. Let $u_{0}$ be an analytic function on a domain $\Omega \subset \mathbb{R}$.

If the formal power series solution (2) of (1) is locally uniformly convergent in $\Omega$, then $u_{0}$ extends to a meromorphic function on $\mathbb{C}$ of the form

$$
u_{0}(z)=2 a z+b+\frac{m}{z}+\sum_{n=1}^{\infty}\left(\frac{1}{z-z_{n}}+\frac{1}{z_{n}}+\frac{z}{z_{n}^{2}}\right),
$$

where $a, b \in \mathbb{C}, m \in \mathbb{N}_{0}$ and $\left\{z_{n}\right\}_{n \in \mathbb{N}} \subset \mathbb{C}^{*} \cup\{\infty\}$ is a sequence with $\left|z_{n}\right|$ nondecreasing (if $z_{n}=\infty$ for some $n \in \mathbb{N}$, then the sum is finite) such that

$$
\sum_{n=1}^{\infty} \frac{1}{\left|z_{n}\right|^{2+\varepsilon}}<\infty \quad \text { for any } \varepsilon>0 .
$$

Conversely, if $u_{0}$ extends to a meromorphic function of the form (7) and (8) holds, then the formal solution (2) of (1) is locally uniformly convergent in $\Omega$.

Proof. The formal power series solution of (1) is given by (2) where $u_{k}$ satisfy the recurrence relations (3). Assume that the formal solution (2) is locally uniformly convergent in $\Omega$. Then it defines a function $u(t, x)$ which is 
analytic on a domain $G \subset \mathbb{R}_{t} \times \mathbb{R}_{x}$ and is a classical solution of (1) in $G\left(^{1}\right)$. Choose $\stackrel{x}{x} \in \Omega$ and define $\varphi$ by (6) for $(t, x) \in G$. The function $\varphi$ is analytic on $G$ and by Lemma 1 it is a solution of

$$
\left\{\begin{array}{l}
\partial_{t} \varphi-\partial_{x}^{2} \varphi=0 \\
\left.\varphi\right|_{t=0}=\varphi_{0}
\end{array}\right.
$$

with $\varphi_{0}(x)=\exp \left\{\int_{\dot{x}}^{x} u_{0}(y) d y\right\}$ for $x \in \Omega$. Note that $\varphi_{0}$ is analytic on $\Omega$ and does not vanish on $\Omega$. On the other hand, the solution of (9) is given by

$$
\varphi(t, x)=\sum_{k=0}^{\infty} \frac{1}{k !} \partial^{2 k} \varphi_{0}(x) t^{k} .
$$

Now the series (10) is locally uniformly convergent in $\Omega$ if and only if for any compact set $K \Subset \Omega$ one can find $C<\infty$ such that

$$
\sup _{x \in K}\left|\partial^{2 k} \varphi_{0}(x)\right| \leq C^{k+1} k !, \quad k \in \mathbb{N}_{0},
$$

or equivalently $\varphi_{0}$ extends to an entire function of exponential order at most 2. By the Hadamard factorization theorem ([13, Ch. XIII, Th. 3.5]),

$$
\varphi_{0}(z)=c z^{m} \exp \left\{a z^{2}+b z\right\} \prod_{n=1}^{\infty}\left(1-\frac{z}{z_{n}}\right) \exp \left\{\frac{z}{z_{n}}+\frac{z^{2}}{2 z_{n}^{2}}\right\},
$$

where $a, b, c \in \mathbb{C}, m \in \mathbb{N}_{0}$ and $\left\{z_{n}\right\}_{n \in \mathbb{N}}, z_{n} \neq 0$, is a sequence of complex zeros of $\varphi_{0}$. Furthermore, (8) holds. Finally, taking the logarithmic derivative we get

$$
u_{0}(z)=\left(\ln \varphi_{0}(z)\right)^{\prime}=2 a z+b+\frac{m}{z}+\sum_{n=1}^{\infty}\left(\frac{1}{z-z_{n}}+\frac{1}{z_{n}}+\frac{z}{z_{n}^{2}}\right),
$$

which concludes the proof of the first part of Theorem 1 .

To prove the second part assume that $u_{0}$ is of the form (7) with $z_{n}$ satisfying (8). Clearly we can assume that $m=0$ (possibly by shifting the $z$ variable). Define $\varphi_{0}$ by

$$
\varphi_{0}(z)=\exp \left\{\int_{0}^{z} u_{0}(y) d y\right\}, \quad z \in \mathbb{C} .
$$

Then by (8) and the Borel product theorem ([13, Ch. XIII, Th. 3.1])

$$
\begin{aligned}
\varphi_{0}(z) & =\exp \left\{a z^{2}+b z\right\} \exp \left\{\sum_{n=1}^{\infty}\left(\ln \left(z-z_{n}\right)+\frac{z}{z_{n}}+\frac{z^{2}}{2 z_{n}^{2}}-\ln \left(-z_{n}\right)\right)\right\} \\
& =\exp \left\{a z^{2}+b z\right\} \prod_{n=1}^{\infty}\left(1-\frac{z}{z_{n}}\right) \exp \left\{\frac{z}{z_{n}}+\frac{z^{2}}{2 z_{n}^{2}}\right\}
\end{aligned}
$$

$\left({ }^{1}\right)$ We can assume that $G$ is $\mathbb{R}_{x}$-convex. 
is an entire function of exponential order at most 2 , which vanishes only at $z_{n}$, $n \in \mathbb{N}$. Since $u_{0}$ was assumed to be analytic on $\Omega$ the points $z_{n}, n \in \mathbb{N}$, do not belong to $\Omega$. Hence the solution (10) of (9) is an analytic function on a domain $G \subset \mathbb{R}_{t} \times \mathbb{R}_{x}$ such that $\{t=0\} \times \Omega \subset G$. Finally, by Lemma 1 the function $u$ defined by (5) is a solution of (1) analytic on $G$.

From the proof of Theorem 1 we obtain a Liouville type theorem for solutions of the Burgers equation.

COROLlary 1. The solution of (1) is an entire function in both variables if and only if it is a constant.

Proof. Indeed, the solution (10) of the heat equation (9) is convergent for all $t \in \mathbb{C}$ if and only if $\varphi_{0}$ is an entire function of exponential order at most 1 . Now the assumption about holomorphy of $u_{0}$ in $z$ implies that it has no poles and so it is a constant.

3. Borel summability. Now we proceed to the characterization of the Borel summable solutions of (1). First of all recall the definition of Borel summability.

Definition ([2, 14]). Let $\Omega$ be a domain in $\mathbb{R}$ and let $\varphi_{n} \in \mathcal{O}(\Omega)$ for $n \in \mathbb{N}_{0}$. We say that a formal power series

$$
\widehat{\varphi}(t, x)=\sum_{n=0}^{\infty} \varphi_{n}(x) t^{n}
$$

is Borel summable (or 1-summable) in a direction $\theta \in \mathbb{R}$ with respect to $t$ locally uniformly in $\Omega$ if the following two conditions hold:

1 . For any $x \in \Omega$ the function

$$
t \mapsto g_{x}(t)=g(t, x)=\sum_{n=0}^{\infty} \frac{\varphi_{n}(x)}{n !} t^{n}
$$

has a radius of convergence $r_{x}>0$, locally uniformly bounded away from zero on $\Omega$.

2. For any $x \in \Omega$ one can find $\delta_{x}>0$ such that $g_{x}(t)$ can be continued analytically to a sector

$$
S\left(\theta, \delta_{x}\right)=\left\{t \in \mathbb{C}^{*}:|\arg (t-\theta)| \leq \delta_{x}\right\}
$$

and for any $\alpha$ with $|\theta-\alpha| \leq \delta_{x}$ one can find $C_{x, \alpha}, L_{x, \alpha}<\infty$ such that

$$
\left|g_{x}(\tau)\right| \leq C_{x, \alpha} e^{L_{x, \alpha}|\tau|} \quad \text { for } \tau \in l_{\alpha}:=e^{i \alpha} \mathbb{R}_{+} ;
$$

furthermore, $1 / \delta_{x}, C_{x, \alpha}$ and $L_{x, \alpha}$ are locally uniformly bounded on $\Omega$ and for $\alpha$ with $|\theta-\alpha| \leq \delta_{x}$. 
In that case the function

$$
f_{x}(t)=f(t, x)=t^{-1} \int_{l_{\alpha}} g(\tau, x) e^{-\tau / t} d \tau, \quad t \in G_{x, \alpha},
$$

is called the Borel sum (or 1-sum) of $\widehat{\varphi}$ in direction $\theta$; here $G_{x, \alpha}=\left\{t \in \mathbb{C}^{*}\right.$ : $\left.|t|^{-1} \cos (\alpha-\arg t)>L_{x, \alpha}\right\}$. Gluing together $f_{x}$ over $\alpha$ with $|\theta-\alpha| \leq \delta_{x}$ we infer that $f_{x}$ is holomorphic in a sectorial neighborhood $G_{x}$ of zero, bisected by $l_{\theta}$ and of opening bigger than $\pi / 2$. Finally, $f(t, x)$ is analytic on $G=\left\{(t, x): x \in \Omega, t \in G_{x}\right\}$. Recall also that the mapping \{Borel summable series $\} \rightarrow\{$ Borel sums $\}$ is an injection.

Note that if (11) is Borel summable in direction $\theta$, then it is also Borel summable in directions $\theta+2 k \pi, k \in \mathbb{Z}$. Furthermore, if (11) is Borel summable in all directions $\theta \in[0,2 \pi)$, then it is convergent.

The Borel summability of formal solutions of the one-dimensional heat equation (9) was characterized by D. A. Lutz, M. Miyake and R. Schäfke [14]. See also [2].

THEOREM LMS. Let $\varphi_{0}$ be an analytic function on a domain $\Omega \subset \mathbb{R}$ and $\theta \in \mathbb{R}$. The formal power series solution $\widehat{\varphi}(t, x)$ of the heat equation (9) is Borel summable in direction $\theta$ if and only if $\varphi_{0}$ extends analytically to a function holomorphic on a domain $D(\theta, \alpha)$ containing a double cone

$$
(\stackrel{\circ}{x}+S(\theta / 2, \alpha)) \cup(\stackrel{\circ}{x}+S(\theta / 2+\pi, \alpha))
$$

with some $\stackrel{\circ}{x} \in \Omega, \alpha>0$ and has in $D(\theta, \alpha)$ at most an exponential growth of order at most 2 .

Now we are ready to state the second main result of the paper.

THEOREM 2. Let $u_{0}$ be an analytic function on a domain $\Omega \subset \mathbb{R}$ and $\theta \in \mathbb{R}$.

If the formal power series solution (2) of (1) is Borel summable in direction $\theta$ locally uniformly in $\Omega$, then $u_{0}$ extends analytically to a function meromorphic on a domain $D(\theta, \alpha)$ containing a double cone $(\stackrel{x}{x} S(\theta / 2, \alpha)) \cup$ $(\stackrel{\circ}{x}+S(\theta / 2+\pi, \alpha))$ with some $\stackrel{\circ}{x} \in \Omega, \alpha>0$ and has in $D(\theta, \alpha)$ at most simple poles with residues in $\mathbb{N}$.

Conversely, if $u_{0}$ extends to a meromorphic function on $D(\theta, \alpha)$ of the form

$$
u_{0}(z)=\frac{m}{z}+\sum_{n=1}^{\infty}\left(\frac{1}{z-z_{n}}+\frac{1}{z_{n}}+\frac{z}{z_{n}^{2}}\right)+v(z), \quad z \in D(\theta, \alpha),
$$

where $m \in \mathbb{N}_{0}, 0 \neq z_{n} \in D(\theta, \alpha)$ satisfy (8), $v$ is holomorphic on $D(\theta, \alpha)$ and $|v(z)| \leq a|z|+b$ for $z \in D(\theta, \alpha)$ with some $a, b<\infty$, then the formal power series solution (2) of (1) is Borel summable in direction $\theta$ locally uniformly in $\Omega$. 
Proof. Assume that the formal solution (2) of (1) is Borel summable in direction $\theta$ locally uniformly in $\Omega$. Then its Borel sum $u(t, x)$ is an analytic function on a domain $G=\left\{(t, x): x \in \Omega, t \in G_{x}\right\}\left({ }^{2}\right)$ and it is a classical solution of (1). Choose $\stackrel{\circ}{x} \in \Omega$ and define $\varphi$ by (6) for $x \in \Omega$ and $t \in G_{x}$. The function $\varphi$ is analytic on $G$ and by Lemma 1 it is a solution of (9) with $\varphi_{0}(x)=\exp \left\{\int_{\check{x}}^{x} u_{0}(y) d y\right\}$ for $x \in \Omega$. Hence the formal solution (10) of (9) is Borel summable to $\varphi$. Now it follows from Theorem LMS that $\varphi_{0}$ extends to a function holomorphic on a domain $D(\theta, \alpha)$ with some $\alpha>0$ and has in $D(\theta, \alpha)$ at most an exponential growth of order at most 2 . Let $m \in \mathbb{N}_{0}$ be the order of zero of $\varphi_{0}$ at the origin and $z_{n} \neq 0, n \in \mathbb{N}$, be zeros of $\varphi_{0}$ in $D(\theta, \alpha)$ counted with their multiplicity. Let $p_{n} \in \mathbb{N}, n \in \mathbb{N}$, be such that for any $R<\infty, \sum_{n=1}^{\infty}\left(R /\left|z_{n}\right|\right)^{p_{n}}<\infty$ (one can put $p_{n}=n$ ). Define

$$
\psi(z)=z^{m} \prod_{n=1}^{\infty}\left(1-\frac{z}{z_{n}}\right) \exp \left\{\frac{z}{z_{n}}+\cdots+\frac{1}{p_{n}}\left(\frac{z}{z_{n}}\right)^{p_{n}}\right\}, \quad z \in \mathbb{C} .
$$

Then $\varphi_{0} / \psi$ is holomorphic in $D(\theta, \alpha)$ and does not vanish there. Hence $\ln \left(\varphi_{0} / \psi\right)$ is holomorphic in $D(\theta, \alpha)$ and so is $\left(\ln \left(\varphi_{0} / \psi\right)\right)^{\prime}=\varphi_{0}^{\prime} / \varphi_{0}-\psi^{\prime} / \psi$. Since $u_{0}=\varphi_{0}^{\prime} / \varphi_{0}$ and

$$
\frac{\psi^{\prime}(z)}{\psi(z)}=\frac{m}{z}+\sum_{n=1}^{\infty}\left\{\frac{1}{z-z_{n}}+\frac{1}{z_{n}}+\cdots+\frac{1}{z_{n}}\left(\frac{z}{z_{n}}\right)^{p_{n}-1}\right\},
$$

we get the conclusion of the first part of Theorem 2 .

To prove the second part assume that $u_{0}$ extends to a meromorphic function on $D(\theta, \alpha)$ of the form (15) with $v$ holomorphic on $D(\theta, \alpha)$ and $|v(z)| \leq a|z|+b$ for $z \in D(\theta, \alpha)$ with some $a, b<\infty$. Clearly we can assume that $m=0$ (by possibly shifting the $z$ variable). Define $\varphi_{0}$ and $w$ by

$$
\varphi_{0}(z)=\exp \left\{\int_{0}^{z} u_{0}(y) d y\right\}, \quad w(z)=\int_{0}^{z} v(y) d y, \quad z \in D(\theta, \alpha) .
$$

Then by (8) and the Borel product theorem,

$$
\varphi_{0}(z)=\exp \{w(z)\} \prod_{n=1}^{\infty}\left(1-\frac{z}{z_{n}}\right) \exp \left\{\frac{z}{z_{n}}+\frac{z^{2}}{2 z_{n}^{2}}\right\}
$$

is holomorphic in $D(\theta, \alpha)$ of exponential growth of order at most 2, which vanishes only at points $z_{n}, n \in \mathbb{N}$. Hence by Theorem LMS the formal solution (10) of (9) is Borel summable in direction $\theta$ to a classical solution $\varphi(t, x)$ of $(9)$ a domain $G$. Finally, by Lemma 1 the function $u$ defined by (5) is a classical solution of (1) on $G \backslash\left\{z_{n}\right\}_{n=1}^{\infty}$ which is a Borel sum in direction $\theta$ of the formal solution (2).

$\left({ }^{2}\right)$ We can assume that $G$ is $\mathbb{R}_{x}$-convex. 
Note that the necessary condition in Theorem 2 for Borel summability of formal solutions of (1) is weaker than the sufficient condition. The difference is caused by the lack of characterization of functions holomorphic on a sector and of exponential growth in terms of density of their zeros. But, inspecting the proof of Theorem 2 we can formulate

COROLlary 2. Let $u_{0}$ be an analytic function on a domain $\Omega \subset \mathbb{R}$ and $\theta \in \mathbb{R}$. The formal power series solution (2) of (1) is Borel summable in the direction $\theta$ if and only if the Cole-Hopf transform of $u_{0}$ extends analytically to a function holomorphic on a domain $D(\theta, \alpha)$ containing a double cone $(\stackrel{\circ}{x}+S(\theta / 2, \alpha)) \cup(\stackrel{\circ}{x}+S(\theta / 2+\pi, \alpha))$ with some $\stackrel{\circ}{x} \in \Omega, \alpha>0$ which has in $D(\theta, \alpha)$ at most an exponential growth of order at most 2 .

REMARK. Note that if $u_{0}$ is meromorphic on the union of domains $D(\theta, \alpha)$ over $0 \leq \theta<\pi$ and has simple poles with residues in $\mathbb{N}$, and at most linear growth at $\infty$, then the formal power series solution of (1) is convergent. This is the case when $u_{0}$ is a rational function on $\mathbb{C}$ and has simple poles with residues in $\mathbb{N}$, and at most linear growth at $\infty$.

4. Examples. In this section we give a few applications of Theorems 1 and 2 .

ExAmple 1. If $u_{0}(x)=a x+b, a \neq 0$, then the solution of (1) is given by

$$
u(t, x)=\frac{a x+b}{1-2 a t}, \quad t \neq \frac{1}{2 a} .
$$

EXAMPLE 2 . Let $u_{0}$ be a rational function vanishing at $\infty$ with simple poles, i.e.

$$
u_{0}(x)=\sum_{i=1}^{n} \frac{c_{i}}{x-a_{i}} \quad \text { with some } a_{i} \in \mathbb{C}, c_{i} \in \mathbb{C}^{*}, i=1, \ldots, n .
$$

If for some $i \in\{1, \ldots, n\}, a_{i}=0$ and $c_{i} \notin \mathbb{N}_{0}$, then the formal solution of (1) is not Borel summable in direction $\theta=0$. Otherwise, the formal solution is Borel summable in directions

$$
\theta \notin \bigcup_{i=1, \ldots, n: a_{i} \neq 0}\left\{2 \arg \left(a_{i}\right)\right\}
$$

and if $a_{i} \neq 0$, then it is Borel summable in direction $\theta=2 \arg \left(a_{i}\right)$ if and only if $c_{j}$ are in $\mathbb{N}$ for all $j$ with $a_{j}=0$ or $\arg a_{j}-\arg a_{i} \in\{0, \pm \pi\}$. Hence the formal solution is convergent if and only if $c_{i}$ are in $\mathbb{N}$ for $i=1, \ldots, n$. In that case the solution can be constructed explicitly. Namely, if (16) holds with all $c_{i}=1$, then the Cole-Hopf transform of $u_{0}$ is

$$
\varphi_{0}(x)=c \prod_{i=1}^{n}\left(x-a_{i}\right) \quad \text { with some } c \in \mathbb{C} .
$$


Denote by $\sigma_{j}(a)$ the $j$ th symmetric function of $a=\left(a_{1}, \ldots, a_{n}\right), j=1, \ldots, n$, i.e. $\sigma_{j}(a)=\sum_{1 \leq i_{1}<\cdots<i_{j} \leq n} a_{i_{1}} \ldots a_{i_{j}}$. Then the solution to the heat equation (9) with data $\varphi_{0}$ is

$$
\begin{aligned}
\varphi(t, x)= & \sum_{k=0}^{\infty} \frac{1}{k !} \partial^{2 k} \varphi_{0}(x) t^{k} \\
= & c \sum_{k=0}^{\infty} \frac{1}{k !} \partial^{2 k}\left(x^{n}-\sigma_{1}(a) x^{n-1}+\cdots+(-1)^{n} \sigma_{n}(a)\right) t^{k} \\
= & c\left(x^{n}-\sigma_{1}(a) x^{n-1}+\cdots+(-1)^{n} \sigma_{n}(a)\right) \\
& +\frac{c}{1 !}\left(n(n-1) x^{n-2}+\cdots+(-1)^{n-2} 2 ! \sigma_{n-2}(a)\right) t \\
& +\cdots+ \begin{cases}\frac{c}{k !}(2 k) ! t^{k} & \text { if } n=2 k, \\
\frac{c}{k !}\left(\frac{(2 k+1) !}{1 !} x-(2 k) ! \sigma_{1}(a)\right) t^{k} & \text { if } n=2 k+1 .\end{cases}
\end{aligned}
$$

So the solution of (1) with the initial data $u_{0}$ is

$$
u(t, x)=\frac{\varphi_{x}^{\prime}(t, x)}{\varphi(t, x)} .
$$

The next two examples are specifications of Example 2.

EXAMPLE 3. Let

$$
u_{0}(x)=\frac{c}{x}, \quad x \neq 0 .
$$

Then the formal solution of $(1)$ is Borel summable in directions $\theta \neq 0 \bmod 2 \pi$ and it is Borel summable in direction $\theta=0$ if and only if $c \in \mathbb{N}_{0}$. If $c=n \in \mathbb{N}$ the solution is, in fact, convergent and is given by

$$
u(t, x)=\frac{n \cdot \sum_{i=0}^{\lfloor(n-1) / 2\rfloor} \frac{(n-1) !}{(n-1-2 i) ! i !} x^{n-1-2 i} t^{i}}{x \cdot \sum_{i=0}^{\lfloor n / 2\rfloor} \frac{n !}{(n-2 i) ! i !} x^{n-1-2 i} t^{i}}
$$

for any $x \neq 0$ and small $|t|$.

EXAMPLE 4. Let

$$
u_{0}(x)=\frac{2 c x}{x^{2}+1}=\frac{c}{x+i}+\frac{c}{x-i}, \quad x \neq \pm i .
$$

Then a formal solution of (1) is Borel summable in directions $\theta \neq \pi \bmod 2 \pi$. It is Borel summable in direction $\theta=\pi$ if and only if $c \in \mathbb{N}_{0}$. In that case it is convergent. In particular,

$$
u(t, x)= \begin{cases}\frac{2 x}{x^{2}+1+2 t} & \text { if } c=1, \\ \frac{4 x\left(x^{2}+1\right)+24 x t}{\left(x^{2}+1\right)^{2}+\left(12 x^{2}+4\right) t+12 t^{2}} & \text { if } c=2 .\end{cases}
$$


EXAMPLE 5. Let

$$
u_{0}(x)=c \tan x \quad \text { for } x \notin \pi / 2+\pi \mathbb{Z} .
$$

Then the formal solution of (1) is Borel summable in directions $\theta \neq 0 \bmod 2 \pi$. The formal solution is Borel summable in direction $\theta=0$ (and hence convergent) if and only if $c \in-\mathbb{N}_{0}$. In particular, $u(t, x)=-e^{-t} \tan x$ if $c=-1$.

ExAmple 6 . Let $\omega_{1}, \omega_{2} \in \mathbb{C}^{*}$ be linearly independent over $\mathbb{R}$ and let $\Omega=\omega_{1} \mathbb{Z}+\omega_{2} \mathbb{Z}$ be a lattice in $\mathbb{C}$. Let

$$
\zeta(z, \Omega)=\frac{1}{z}+\sum_{0 \neq \omega \in \Omega}\left(\frac{1}{z-\omega}+\frac{1}{\omega}+\frac{z}{\omega^{2}}\right)
$$

be the Weierstrass $\zeta$-function. Then the formal power series solution of (1) with $u_{0}(x)=c \zeta(x, \Omega)$ is Borel summable in a direction $\theta$ if and only if $c \in \mathbb{N}_{0}$. Then it is in fact convergent. The same conclusion holds if $u_{0}(x)=$ $c \zeta(x-a, \Omega)$ with any $a \in \mathbb{C}$ since $\bigcup_{\omega \in \Omega}\{\arg (\omega-a)\}$ is a dense set in $0 \leq \theta<2 \pi$.

Remark. D. Senouf [19] obtained the solution of (1) with $u_{0}(x)=$ $-2 x^{3}+x / c, c>0$, in the form of a Mittag-Leffler expansion.

Acknowledgements. Most of the work on this paper was done during the author's stay at the Laboratoire Jacques-Louis Lions, Université Pierre et Marie Curie in Paris, in March through May, 2008. The author would like to express his gratitude to T. Cazenave and A. Haraux for hospitality and enlightening discussions.

This research was partially supported by EC FP6 MCA-ToK program SPADE2, MTKD-CT-2004-014508 and Polish MNiSW SPB-M.

\section{References}

[1] W. Balser, Multisummability of formal power series solutions of partial differential equations with constant coefficients, J. Differential Equations 201 (2004), 63-74.

[2] - Divergent solutions of the heat equation: on an article of Lutz, Miyake and Schäfke, Pacific J. Math. 188 (1999), 53-63.

[3] W. Balser and S. Malek, Formal solutions of the complex heat equation in higher spatial variables, in: Proceedings of Global and Asymptotic Analysis of Differential Equations in the Complex Domain, Kôkyûroku RIMS 1367 (2004), 87-94.

[4] D. Bekiranov, The initial-value problem for the generalized Burgers' equation, Differential Integral Equations 9 (1996), 1253-1265.

[5] E. R. Benton and G. W. Platzman, A table of solutions of the one-dimensional Burgers equation, Quart. Appl. Math. 30 (1972), 195-212.

[6] J. M. Burgers, The mathematical model illustrating the theory of turbulence, Adv. Appl. Mech. 1 (1948), 171-199.

[7] D. B. Dix, Nonuniqueness and uniqueness in the initial-value problem for Burgers' equation, SIAM J. Math. Anal. 27 (1996), 708-724. 
[8] H. Chen, Z. Luo and C. Zhang, On the summability of formal solutions for a class of nonlinear singular PDEs with irregular singularity, in: Recent Progress on Some Problems in Several Complex Variables and Partial Differential Equations, Contemp. Math. 400, Amer. Math. Soc., Providence, RI, 2006, 53-64.

[9] H. Chen and Z. Zhang, On the Borel summability of formal solutions for some first order singular PDEs with irregular singularity, Acta Math. Sci. Ser. B Engl. Ed. 25 (2005), 419-426.

[10] J. D. Cole, On a quasi-linear parabolic equation occurring in aerodynamics, Quart. Appl. Math. 9 (1951), 225-236.

[11] E. Hopf, The partial differential equation $u_{t}+u u_{x}=\mu u_{x x}$, Comm. Pure Appl. Math. 3 (1950), 201-230.

[12] K. T. Joseph and P. L. Sachdev, Initial boundary value problems for scalar and vector Burgers equations, Stud. Appl. Math. 106 (2001), 481-505.

[13] S. Lang, Complex Analysis, Grad. Texts in Math. 103, Springer, New York, 1999.

[14] D. A. Lutz, M. Miyake and R. Schäfke, On the Borel summability of divergent solutions of the heat equation, Nagoya Math. J. 154 (1999), 1-20.

[15] G. Eysik, Formal solutions of Burgers type equations, Funct. Approx., submitted.

[16] S. Michalik, Summability of divergent solutions of the $n$-dimensional heat equation, J. Differential Equations 229 (2006), 353-366.

[17] S. Ouchi, Multisummability of formal solutions of some linear partial differential equations, ibid. 185 (2002), 513-549.

[18] - Multisummability of formal power series solutions of nonlinear partial differential equations in complex domains, Asymptotic Anal. 47 (2006), 187-225.

[19] D. Senouf, Dynamics and condensation of complex singularities for Burgers' equation. I, SIAM J. Math. Anal. 28 (1997), 1457-1489.

Institute of Mathematics

Polish Academy of Sciences

P.O. Box 21

00-956 Warszawa 10, Poland

and

Jan Kochanowski University, Kielce, Poland

E-mail: lysik@impan.pl

Received 18.9.2008

and in final form 6.1.2009 